Classification

Physics Abstracts

$21.10 \mathrm{H}-27.30$

\title{
Low lying yrast states of ${ }^{32} \mathrm{~S}$
}

\author{
R. M. Freeman, F. Haas, C. Beck and A. Niemeskern \\ Centre de Recherches Nucléaires et Université Louis Pasteur, 67037 Strasbourg Cedex, France
}

(Reçu le 20 janvier 1983, révisé le 1er avril, accepté le 12 avril 1983)

\begin{abstract}
Résumé. - Des mesures de corrélations angulaires pour la réaction ${ }^{29} \mathrm{Si}(\alpha, \mathrm{n} \gamma){ }^{32} \mathrm{~S}$ ont permis d'attribuer $J^{\pi}=5^{-}$au niveau à $6762-\mathrm{keV}$. Dans le noyau ${ }^{32} \mathrm{~S}$, quatre nouveaux niveaux ont été observés à des énergies d'excitation de 7 567-, 8 345-, $9464-$ et $9637-\mathrm{keV}$. Une attribution $J^{\pi}=5^{+}$ a été faite pour le niveau à $7567-\mathrm{keV}$ et le niveau à $8345-\mathrm{keV}$ est un excellent candidat pour être le premier état $6^{+}$dans ${ }^{32} \mathrm{~S}$. Les deux niveaux d'énergie d'excitation supérieure à $9 \mathrm{MeV}$ sont probablement des niveaux de parité négative et de moment angulaire élevé.
\end{abstract}

\begin{abstract}
From angular correlation measurements with the ${ }^{29} \mathrm{Si}(\alpha, \mathrm{n} \gamma){ }^{32} \mathrm{~S}$ reaction the $6762-\mathrm{keV}$ level has been assigned $J^{\pi}=5^{-}$. Four new levels of ${ }^{32} S$ have been observed at $E_{x}=7567-, 8345$-, 9464 - and $9637-\mathrm{keV}$. The $7567-\mathrm{keV}$ level has been assigned $J^{\pi}=5^{+}$and the $8345-\mathrm{keV}$ level is a good candidate for the lowest $6^{+}$state. The two higher levels are probably negative parity high spin states.
\end{abstract}

In the course of experiments on $A=32$ nuclei we have observed a number of levels of ${ }^{32} \mathrm{~S}$ for which the $\gamma$-ray decay modes suggest spin assignments $J \geqslant 5$. The yrast states of ${ }^{32} \mathrm{~S}$ are poorly known and in a recent compilation [1] no definite assignments $J>4$ were given though the spin and parity of most states to above $E_{x}=7 \mathrm{MeV}$, including the first three states of figure 1, have been firmly established. We are therefore presenting in this letter our angular correlation data for these transitions though a fuller account will be given with other results on completion of the present series of experiments.

The ${ }^{32} \mathrm{~S}$ levels were excited by the ${ }^{29} \mathrm{~S}(\alpha, \mathrm{n})$ reaction using the $\mathrm{He}^{++}$beam from the Strasbourg CN Van de Graaff accelerator. We will be concerned in this letter with the $\gamma$ transitions indicated in figure 1 . These were observed in the present experiments in $n-\gamma$ coincidence spectra and their place in the decay scheme was verified in supplementary $\gamma-\gamma$ coincidence experiments. The four highest levels in figure 1 had not been previously reported. The two transitions from the $6762-\mathrm{keV}$ level have already been observed and studied by the ${ }^{31} \mathbf{P}(\mathrm{p}, \gamma)$ reaction [1] but no unambiguous spin assignment and only a lower limit for the lifetime ( $>300 \mathrm{fs}$ ) of the state had yet been made. The line shape that we have observed for these two transitions in the $n-\gamma$ coincidence spectra indicates a lifetime of the order of 400 to $500 \mathrm{fs}$. The transitions from the four higher levels were all strongly Doppler shifted consistent with lifetimes substantially shorter than that for the $6762-\mathrm{keV}$ level.

To determine spins for these levels $\mathrm{n}-\gamma$ angular correlations were measured in a collinear geometry at a bombarding energy $E_{\alpha}=12 \mathrm{MeV}$ for the 6762 - and $7567-\mathrm{keV}$ levels and at $E_{\alpha}=$ 


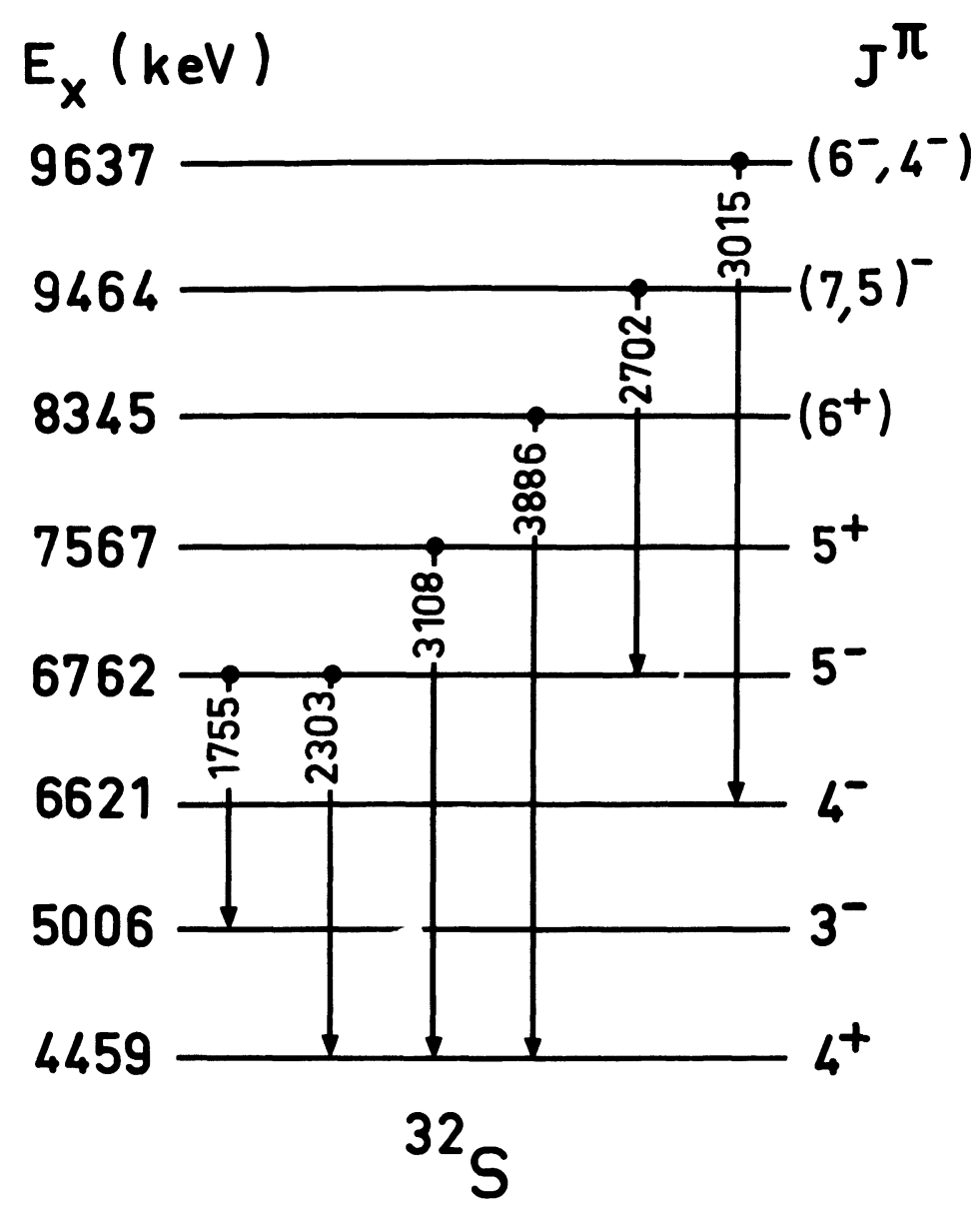

Fig. 1. - The transitions of ${ }^{32} \mathrm{~S}$ studied in the present work. $J^{\pi}$ values for the first three states are from reference [1]. For the other states firm or probable assignments are given on the basis of the present measurements as discussed in the text. The four highest states are new levels which have not been previously reported.

$14 \mathrm{MeV}$ for the three higher states. The neutrons were detected at $0^{\circ}$ to the beam direction and the $\gamma$-rays in large volume $\mathrm{Ge}(\mathrm{Li})$ detectors placed at angles ranging between $90^{\circ}$ to $158^{\circ}$. The correlations were fitted with Legendre polynomials up to order 4 and the results, corrected for the finite size of the $\mathrm{Ge}(\mathrm{Li})$ detectors, are listed in table I. The final column in table I gives the possible spin values consistent with the angular correlations and lifetimes. For the new levels the measured transition energies are also given.

The angular correlations measured at $E_{\alpha}=12 \mathrm{MeV}$ have permitted us to assign unique $J^{\pi}$ values to both the $6762-$ and $7567-\mathrm{keV}$ levels. Previously the values for the $6762-\mathrm{keV}$ level had been limited to $\mathrm{J}^{\pi}=3^{-}, 4^{+}, 5^{-}$. With the present results only $5^{-}$has been retained. For this spin good fits were obtained with mixing ratios essentially equal to zero for both the $1755-\mathrm{keV}$ (E2) and the $2303-\mathrm{keV}$ (E1) transitions. The correlation for the $3108-\mathrm{keV}$ transition from the $7567-\mathrm{keV}$ level is characterized by a large positive $a_{4} / a_{0}$ coefficient which could only be fitted assuming an almost pure quadrupole transition from a $J=5$ level. The quadrupole nature of the transition implies positive parity for the state. The experimental angular correlations for these two states are shown in figure 2 together with the best fits obtained assuming pure multipolarities for the three transitions. 


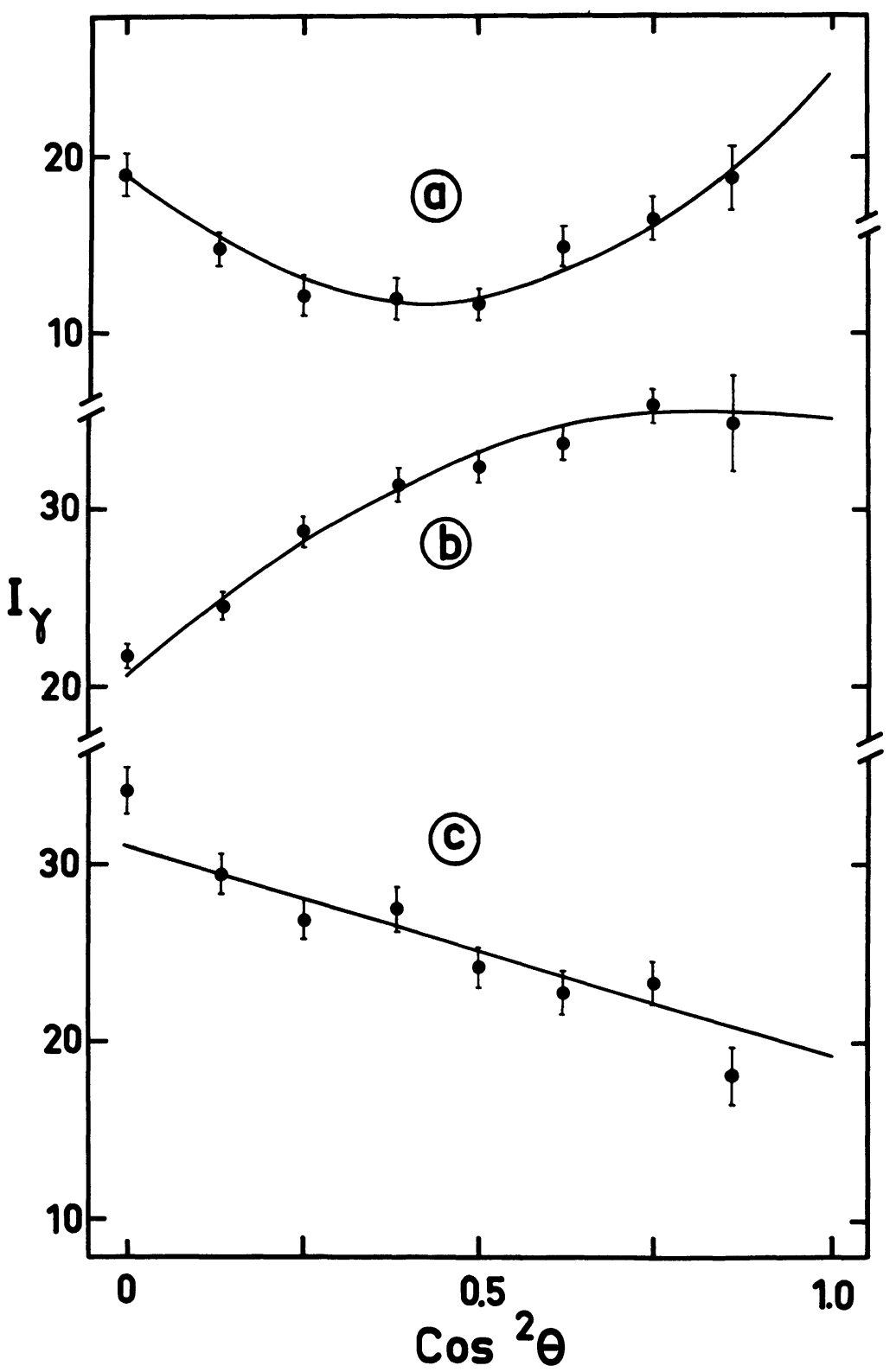

Fig. 2. - Angular correlations measured for the 7567 - and $6762-\mathrm{keV}$ levels : (a) $7567\left(5^{+}\right) \rightarrow 4459\left(4^{+}\right)$ transition; (b) $6762\left(5^{-}\right) \rightarrow 5006\left(3^{-}\right)$transition; (c) $6762\left(5^{-}\right) \rightarrow 4459\left(4^{+}\right)$transition. The full lines are best fits assuming pure E2 for $(a)$ and $(b)$ and pure E1 for $(c)$. 
Table I. - Coefficients for the Legendre polynomial fits to the n- $\gamma$ angular correlations of the ${ }^{29} \mathrm{Si}(\alpha, \mathrm{n} \gamma){ }^{32} \mathrm{~S}$ reaction

\begin{tabular}{|c|c|c|c|c|c|}
\hline$E_{x}(\mathrm{keV})$ & $E_{\gamma}(\mathrm{keV})$ & $a_{2} / a_{0}$ & $a_{4} / a_{0}$ & $J_{\mathrm{f}}^{\pi}$ & $J_{\mathrm{i}}^{\pi}$ \\
\hline 6762 & 1755 & $+0.40 \pm 0.04$ & $-0.15 \pm 0.05$ & $3^{-}$ & $5^{-}$ \\
& 2303 & $-0.36 \pm 0.05$ & $+0.07 \pm 0.08$ & $4^{+}$ & \\
7567 & $3108.4 \pm 1.2$ & $+0.07 \pm 0.08$ & $+0.67 \pm 0.10$ & $4^{+}$ & $5^{+}$ \\
8345 & $3886.1 \pm 1.5$ & $+0.30 \pm 0.07$ & $-0.20 \pm 0.09$ & $4^{+}$ & $6^{+}-2^{+}, 4^{-}$ \\
9464 & $2702.1 \pm 1.5$ & $+0.34 \pm 0.07$ & $-0.39 \pm 0.09$ & $5^{-}$ & $7^{-}, 5^{-}$ \\
9637 & $3015.3 \pm 1.5$ & $+0.38 \pm 0.09$ & $-0.37 \pm 0.11$ & $4^{-}$ & $6^{-}, 4^{-}\left(3^{-}, 4^{+}, 5^{-}\right)$ \\
\hline
\end{tabular}

The correlations measured at $E_{\alpha}=14 \mathrm{MeV}$ for the three higher states all show forms typical of stretched E2 transitions, suggesting $J^{\pi}=6^{+}, 7^{-}$and $6^{-}$for the $8345-, 9464$ - and $9637-\mathrm{keV}$ levels respectively. But other spin assignments cannot be excluded and in particular the correlations can also be fitted under the conditions $J_{\mathrm{i}}=J_{\mathrm{f}}$ and $\delta \approx-1.2$. In the case of the $8345-\mathrm{keV}$ level the angular correlation was not strong enough to eliminate any reasonable spin assignment. However $6^{+}$is considered to be most likely as the first $6^{+}$state of ${ }^{32} \mathrm{~S}$ is expected to lie in the region covered by the present experiments. The decay mode and angular correlation for the $8345-\mathrm{keV}$ level are those expected for such a state and no other possible candidate has been observed. There was some preference in favour of $J^{\pi}=5^{-}$for the $9464-\mathrm{keV}$ level from the $\chi^{2}$ fits to the angular correlation, though an acceptable fit was also obtained assuming $7^{-}$. It is noted that there is evidence for a $7^{-}$state at an even lower excitation energy in the neighbouring nucleus ${ }^{34} \mathrm{~S}$ [2]. For the 9 637-keV level the most probable assignments are $6^{-}$and $4^{-}$. Three other values included between brackets in table I correspond to assignments which are less likely but cannot be excluded with a high degree of probability.

Much of the spectroscopic information on ${ }^{32} \mathrm{~S}$ levels in this region comes from capture and light-ion transfer reactions. These would not readily excite the high spin states and this is probably the reason why four of the five levels considered here had not been observed previously. Work on heavy-ion reactions feeding the high spin states of ${ }^{32} \mathrm{~S}$ is lacking. Some heavy-ion $\alpha$-transfer reactions to ${ }^{32} \mathrm{~S}$ have been studied (see, for example, ${ }^{28} \mathrm{Si}\left({ }^{6} \mathrm{Li}, \mathrm{d}\right){ }^{32} \mathrm{~S}$ by Tanabe et al. [3]), but there is little evidence that these reactions feed the same levels seen in the present experiments. The prominent peak which is observed at $E_{x}=8.5 \mathrm{MeV}$ is significantly higher than where we propose a $6^{+}$state and in a region where only low spin states are known.

\section{References}

[1] Endt, P. M. and VAN Der leun, C., Nucl. Phys. A 310 (1978) 1.

[2] Grawe, H. and Kändler, K., Proc. Int. Conf. on Nuclear Structure, Tokyo (1977) 217.

[3] Tanabe, T., Yasue, M., Sato, K., Ogino, K., Kadota, Y., Taniguchi, Y., Obori, K., Makino, K. and Tochi, M., Phys. Rev. C 24 (1981) 2556. 